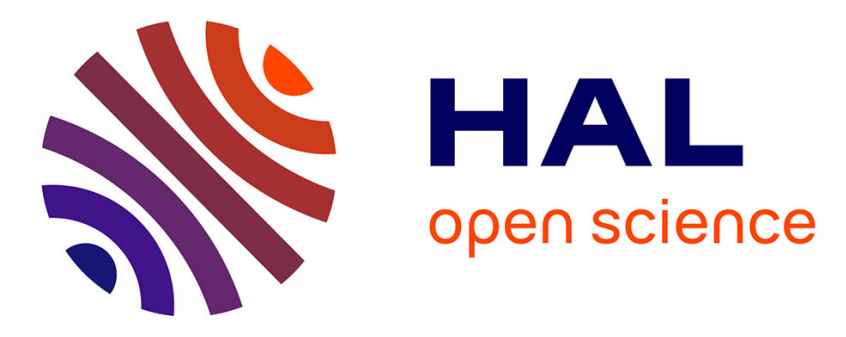

\title{
Stereomotion Processing in the Nonhuman Primate Brain
}

Yseult Héjja-Brichard, Samy Rima, Emilie Rapha, Jean-Baptiste Durand, Benoit Cottereau

\section{- To cite this version:}

Yseult Héjja-Brichard, Samy Rima, Emilie Rapha, Jean-Baptiste Durand, Benoit Cottereau. Stereomotion Processing in the Nonhuman Primate Brain. Cerebral Cortex, 2020, 30 (8), pp.4528-4543. 10.1093/cercor/bhaa055 . hal-03014992v1

\section{HAL Id: hal-03014992 \\ https://hal.science/hal-03014992v1}

Submitted on 19 Nov 2020 (v1), last revised 14 Dec 2020 (v2)

HAL is a multi-disciplinary open access archive for the deposit and dissemination of scientific research documents, whether they are published or not. The documents may come from teaching and research institutions in France or abroad, or from public or private research centers.
L'archive ouverte pluridisciplinaire HAL, est destinée au dépôt et à la diffusion de documents scientifiques de niveau recherche, publiés ou non, émanant des établissements d'enseignement et de recherche français ou étrangers, des laboratoires publics ou privés. 


\title{
Stereomotion Processing in the Nonhuman Primate Brain
}

\author{
Yseult Héjja-Brichard@1,2, Samy Rima ${ }^{1,2}$, Emilie Rapha ${ }^{1,2}$ and \\ Jean-Baptiste Durand ${ }^{1,2}$ and Benoit R. Cottereau ${ }^{1,2}$
}

\author{
${ }^{1}$ Centre de Recherche Cerveau et Cognition, Université de Toulouse, 31052 Toulouse, France and ${ }^{2}$ Centre \\ National de la Recherche Scientifique, 31055 Toulouse, France
}

Address correspondence to Yseult Héjja-Brichard, Centre de Recherche Cerveau et Cognition, Université de Toulouse, 31052 Toulouse, France. Email: yseult.hejja@cnrs.fr; Benoit R Cottereau, Centre National de la Recherche Scientifique, 31055 Toulouse, France. Email: benoit.cottereau@cnrs.fr

\begin{abstract}
The cortical areas that process disparity-defined motion-in-depth (i.e., cyclopean stereomotion [CSM]) were characterized with functional magnetic resonance imaging (fMRI) in two awake, behaving macaques. The experimental protocol was similar to previous human neuroimaging studies. We contrasted the responses to dynamic random-dot patterns that continuously changed their binocular disparity over time with those to a control condition that shared the same properties, except that the temporal frames were shuffled. A whole-brain voxel-wise analysis revealed that in all four cortical hemispheres, three areas showed consistent sensitivity to CSM. Two of them were localized respectively in the lower bank of the superior temporal sulcus ( $\left.\mathrm{CSM}_{\mathrm{STS}}\right)$ and on the neighboring infero-temporal gyrus $\left(\mathrm{CSM}_{\mathrm{ITG}}\right)$. The third area was situated in the posterior parietal cortex $\left(\mathrm{CSM}_{\mathrm{PPC}}\right)$. Additional regions of interest-based analyses within retinotopic areas defined in both animals indicated weaker but significant responses to CSM within the MT cluster (most notably in areas MSTv and FST). Altogether, our results are in agreement with previous findings in both human and macaque and suggest that the cortical areas that process CSM are relatively well preserved between the two primate species.
\end{abstract}

Key words: depth, fMRI, motion, nonhuman primate

\section{Introduction}

Motion perception is a fundamental property of the visual system in most animal species. It enables to track over time the position of elements in a scene and thereby facilitates navigation or interactions with moving objects. Historically, numerous studies have characterized planar (i.e., 2D) motion processing in the primate nervous system (see Maunsell and Newsome 1987). In macaque, single-cell recordings showed that it is computed at the cortical level within an extended network that begins in the primary visual cortex and includes higher level visual areas such as area V4 in the ventral patway (Li et al. 2013) and area V3A (Galletti et al. 1990) or V6 (Pitzalis et al. 2013) in the dorsal pathway. Within the superior temporal sulcus (STS), area MT notably hosts neurons whose responses are highly selective to motion direction (see Maunsell and Newsome 1987) and also reflects motion perception (Newsome and Paré 1988; Britten et al. 1996). In human, neuroimaging studies suggested that $2 \mathrm{D}$ motion is also processed within an extended network that includes a putative homologue of area MT: hMT (Huk et al. 2002). Over the last 20 years, the emergence of monkey fMRI (see Vanduffel et al. 2001) made possible the further characterization of the correspondence between the cortical areas involved in motion processing in the two species. This comparative approach revealed that 2D motion engaged largely similar networks in macaques and in humans. It notably suggested that motion processing in MT and its satellite areas (V4t, FST, and MSTv) on the one hand and in hMT and its satellites (pV4t, pFST, and pMSTv) on the other hand could be homologous (Kolster et al. 2009, 2010). Yet, functional differences between both species have also been documented, since sensitivity to motion in area V3A and 
other regions within the intraparietal sulcus (IPS) was found to be more pronounced in human than in macaque (see Orban et al. 2003 for a review). For instance, cortical areas responsive to motion-defined structures are encountered in the human IPS but not in its monkey counterpart (Vanduffel et al. 2002).

Rather surprisingly, much less is known about the neural mechanisms that process motion along the depth dimension in primates. Motion in depth is nonetheless a very common and important component of motion in everyday life as it can notably signal objects moving towards the head and/or the body. Its estimation derives from two binocular cues: The change of disparity over time (CDOT), which tracks dynamic increase or decrease in horizontal disparity, and the interocular velocity difference (IOVD), based on opponent motion vectors between the two eyes (Nefs et al. 2010). The characterization of the cortical areas processing each of these cues in both human and macaque would lead to a better understanding of how the 3D motion of objects is integrated in the primate brain and to further establish the similarities but also the differences between motion processing in the two species. Over the last 10 years, this important line of research inspired a growing number of studies based on neuroimaging measurements in human and single-cell recordings in macaque.

In human, a pioneer fMRI study (Likova and Tyler 2007) found that the strongest responses to CDOT arise in a cortical region anterior to the hMT+ complex. This region had reduced responses to 2D motion and was labeled "CSM," for cyclopean stereomotion, by the authors. It might therefore be specialized in processing motion-in-depth. From additional analyses within independently defined regions of interest (ROIs), this study also found that several visual areas, such as V3A, V4, and hMT+, were more responsive to CDOT than to static disparity planes. Two years later, Rokers et al. (2009) found specific responses to CDOT and IOVD in the hMT+ complex, but also in area V3A and in lateral occipital regions (LO1/LO2). However, this study did not investigate activations in regions anterior to the hMT+ complex. More recently, Kaestner et al. (2019) confirmed that the hMT+ complex and two groups of ROIs that respectively gathered dorsal (V3A/B and IPS-0) and ventral (V4, LO-1 and LO-2) areas were more responsive to CDOT than to a control condition where the temporal frames were scrambled. These authors also found significant responses to CDOT in the CSM area but that were not as pronounced as those measured in $\mathrm{hMT}+$. Altogether, these three human studies found consistent patterns of cortical responses to CDOT even though the precise functional role of area CSM, and notably how its responses to both 2D and 3D motion differ from those measured in hMT+, remains to be better understood.

In macaque, explorations of motion in depth selectivity at the single-cell level began with electrophysiological recordings in area MT, notably on the grounds that this area has similar 2D motion responses in human and nonhuman primates. Two studies found that MT neurons were selective to motion in depth (Czuba et al. 2014; Sanada and DeAngelis 2014) but that this selectivity was primarily driven by the IOVD cue, with only a small contribution from the CDOT cue (Sanada and DeAngelis 2014). Based on the human findings, it is possible that stronger responses to CDOT could be found in other satellite regions of the MT cluster (e.g., in FST or MSTv) and/or in more anterior regions of the STS. It is also possible that significant responses to CDOT exist in other regions of the ventral and dorsal visual pathways. In order to clarify which regions of the macaque cortex should be explored to better understand the neural mechanisms underlying motion-in-depth processing in primate, it is essential to first determine whether the areas that were identified in human from neuroimaging measurements could have putative homologues in macaques by using a similar approach.

In the present study, we performed fMRI recordings in awake behaving macaques to identify the cortical regions sensitive to motion-in-depth defined by changing disparity over time (CDOT). To facilitate the comparison with previous human data, we used an experimental protocol that was directly inspired from the neuroimaging studies described above (Likova and Tyler 2007; Rokers et al. 2009; Kaestner et al. 2019). In order to precisely determine the limits of the MT cluster, we ran an additional retinotopic mapping experiment that permitted to delineate its four constituting areas V4t, MT, MSTv, and FST (Kolster et al. 2009). This approach allowed us to clarify whether the strongest responses to CDOT in macaque emerge beyond this cluster or within one or several of its components. It also permitted to document CDOT responses in retinotopic area of the early visual cortex (V1, V2, and V3) and along the dorsal (V3A) and ventral (V4) pathways. To determine whether regions activated by CDOT were also responsive to 2D motion, we ran a last experiment where we characterized the responses to moving versus static objects.

\section{Materials and Methods}

\section{Subjects}

Two female rhesus macaques (age: 15-17 years; weight: 5.4$6.2 \mathrm{~kg}$ ) were involved in the study. Animal housing, handling, and all experimental protocols (surgery, behavioral training, and MRI recordings) followed the guidelines of the European Union legislation (2010/63/UE) and of the French Ministry of Agriculture (décret 2013-118). All projects were approved by a local ethics committee (CNREEA code: C2EA-14) and received authorisation from the French Ministry of Research (MP/03/34/10/09). Details about the macaques' surgical preparation and behavioral training are provided elsewhere (Cottereau et al. 2017).

\section{Data Availability}

Data and analysis code will be made available after acceptance of the paper on a dedicated platform (OSF: https://osf.io/yxrsv/).

\section{Experimental Design}

Our stimuli were derived from those of previous fMRI studies that investigated how CSM (motion-in-depth based on CDOT) is processed in humans (Likova and Tyler 2007; Rokers et al. 2009; Kaestner et al. 2019). Our aim was to facilitate the comparison between the cortical networks involved in the two species. We used dynamic random-dot stereograms (dRDS) located within a disk (11 degrees of radius) and refreshed at $30 \mathrm{~Hz}$. The dot density was $15 \%$. To manipulate binocular disparity between the two retinal projections, dots were green in one eye and red in the other and stimuli were observed through red-green anaglyph filters (stimulus code made available on OSF: https://osf.io/yxrsv/). In the CSM condition, dots within the upper and lower parts of the disk changed their disparity in opposition of phase, following a triangular function $(1 \mathrm{~Hz}$ ) between \pm 23.3 arcmin (see Fig. 1A). This disparity range was chosen to maximize the cortical responses to binocular disparities (see Backus et al. 2001; Cottereau et al. 2011). The opposition of phase between stereomotion of the dots in the upper and lower parts of the disk 
led to an average disparity across the visual field of zero at each frame and thereby prevented stimulus-induced change in vergence eye movement. Note that in this condition, motion in depth is defined from the change of disparity over time (CDOT). We chose to use the term CSM in reference to the original human fMRI study of Likova and Tyler (2007).

Our CSM stimulus led to perceive two planes continuously moving alongside a horizontal axis in opposite directions, one plane being located in front of the point of fixation and the other behind the fixation point. The control stimulus consisted of a "temporally scrambled" version (TS) of the CSM stimulus. To create this temporally scrambled condition, we shuffled the frames from the CSM stimulus in order to disrupt the temporal sequence and thus, the motion in depth. Importantly, both conditions were monocularly identical and contained the same disparity distributions. The average relative disparities between dots in the upper versus lower parts of the disk were also identical between our two conditions.

\section{MRI Recordings}

Image Acquisition: Templates of Reference and Functional Sessions Whole-brain images were acquired on a 3 Tesla MR scanner (Phillips Achieva) using a custom 8-channel phased-array coil (RapidBiomed) specifically designed to fit the skull of our macaques while preserving their field of view. Four T1-weighted anatomical volumes were acquired prior to the study for each monkey at a high resolution (MPRAGE; repetition time, $\mathrm{TR}=10.3 \mathrm{~ms}$; echo time, $\mathrm{TE}=4.6 \mathrm{~ms}$, flip angle $=8^{\circ}$; FOV: $155 \times 155 \mathrm{~mm}$; matrix size: $312 \times 192 \mathrm{~mm}$; voxel size $=0.5 \times 0.5 \times 0.5 \mathrm{~mm} ; 192$ sagittal slices acquired in an interleaved order), as well as 300 functional volumes (gradient-echo EPI; TR $=2000 \mathrm{~ms}, \mathrm{TE}=30 \mathrm{~ms}$, flip angle $=75^{\circ}$, SENSE factor $=1.6$; FOV: $100 \times 100 \mathrm{~mm}$; matrix size: $68 \times 64 \mathrm{~mm}$; voxel size $=1.25 \times 1.25 \times 1.5 \mathrm{~mm}, 32$ axial slices acquired in an interleaved order with a thickness of $1.5 \mathrm{~mm}$ and no gap). Those data were recorded in a single session while the macaques were slightly anesthetized (Zoletil 100:10 mg/kg and Domitor: $0.04 \mathrm{mg} / \mathrm{kg}$ ) and their constants monitored with an MR compatible oximeter. Those volumes were then used to create individual anatomical and functional templates of reference.

Our $\mathrm{T}_{2}{ }^{*}$-weighted functional images were acquired with a gradient-echo EPI sequence with interleaved slice acquisition $\left(\mathrm{TR}=2000 \mathrm{~ms}, \mathrm{TE}=30 \mathrm{~ms}\right.$, flip angle $=75^{\circ}$, SENSE factor=1.6; FOV: $100 \times 100 \mathrm{~mm}$; matrix size: $68 \times 64 \mathrm{~mm}$; voxel size $=1.25 \times 1.25 \times 1.5 \mathrm{~mm}, 32$ axial slices acquired in an interleaved order with a thickness of $1.5 \mathrm{~mm}$ and no gap).

\section{Scanning Procedure}

During the functional recording sessions, macaques were headfixed and seated in a sphinx position in a dedicated primate chair (see Fig. 1B). They had to maintain their gaze within a central fixation window $\left(2^{\circ} \times 2^{\circ}\right)$ during daily sessions of up to $2 \mathrm{~h}$. Their fixation was monitored with an ASLC infrared video-based eye tracking setup at $60 \mathrm{~Hz}$ and they were rewarded through a liquid delivery system (Crist Instrument) at intervals whose frequency depended on their fixation performance. Our CSM stimuli were video-projected using a $23^{\circ} \times 23^{\circ}$ field of view (viewing distance $=85 \mathrm{~cm}$ ). We used a blocked design based on cycles within which our two conditions (CSM and TS) were interleaved with baseline periods of fixation (see Fig. 1C). Both the condition and baseline blocks lasted $18 \mathrm{~s}$ (9 TRs) and a cycle was therefore 72-s long (36 TRs). Each run contained three repetitions of this cycle plus an extra baseline that was added at the end for a total duration of 117 TR (234 s). We displayed the stimuli, controlled for the delivery of the liquid reward and the fixation performance using the EventIDE software (Okazolab ${ }^{\circledR}$ ).

\section{Data Analysis}

Templates of Reference

Anatomical and functional templates of reference were created for each individual with the volumes acquired prior to the current study. The anatomical template was obtained with the four $\mathrm{T}_{1}$-weighted anatomical volumes being realigned, averaged, and then coregistered on the MNI space of the 112RM-SL template (McLaren et al. 2009, 2010). To create the functional template, the 300 functional volumes (GE-EPI) were realigned, averaged, and then coregistered on the anatomical template. Both the T1 and the EPI mean images were segmented separately in order to obtain tissue probability maps for the gray matter, the white matter, and the cerebrospinal fluid. These probability maps were used to estimate the normalization parameters from functional (mean EPI) to structural (mean $\mathrm{T}_{1}$ ) images for each individual.

Preprocessing of the Raw Functional Data

Preprocessing and volume-based analyses were carried out with SPM12 in the MATLAB environment (MathWorks ${ }^{\circledR}$ ). Only runs with central gaze fixation above $85 \%$ were kept for further analysis. In total, we kept 43 and 60 runs for both macaques, respectively. The 4 first volumes of each run were discarded (dummy scans) to account for the establishment duration of the BOLD steady-state response. Preprocessing was performed for each volume, run by run. Slice-timing correction was performed first using as a reference the slice acquired in the middle of the acquisition of each TR. Images were then reoriented, coregistered with the EPI template, and transformed to fit the individual $\mathrm{T}_{1}$ template (with the normalization parameters estimated between the mean EPI and $T_{1}$ images; see the Templates of Reference). No motion correction was applied to the images. Finally, the images were smoothed with a spatial Gaussian kernel (FWHM $=2 \times 2 \times 2 \mathrm{~mm}$ ).

\section{HRF Estimation}

Prior to our statistical analyses, we used independent datasets to characterize the BOLD haemodynamic impulse response functions (HRF) separately for each animal. These datasets respectively contained 16 (M01) and 12 (M02) 204-s-long runs that consisted of 6 cycles of 4 -s full field counter phasing $(10 \mathrm{~Hz})$ checkerboards separated by a 30-s blank interval (see more details about this procedure in Cottereau et al. 2017). Data were preprocessed using the pipeline described above and projected onto individual surfaces generated with the CARET software (Van Essen et al. 2001). Following Dumoulin and Wandell's procedure (Dumoulin and Wandell 2008), we extracted the BOLD responses from nodes within the anatomically defined V1 of each individual. We only kept visually responsive nodes, that is, those whose signal-to-noise ratio (SNR) was greater than 3 . This SNR was estimated with a Fourier analysis of the average time courses across runs where the signal corresponded to the Fourier coefficient amplitude at the stimulation frequency $F$ (i.e., $F=1 / 34$ ) and the noise was given by the average moduli at the two neighboring frequencies (i.e., $F-\delta f$ and $F+\delta f$, where $\delta f=1 / 2$ is the resolution of our frequency analysis). We computed the average time course of these nodes during one cycle and used this average time course for estimating the HRF. The HRF was 


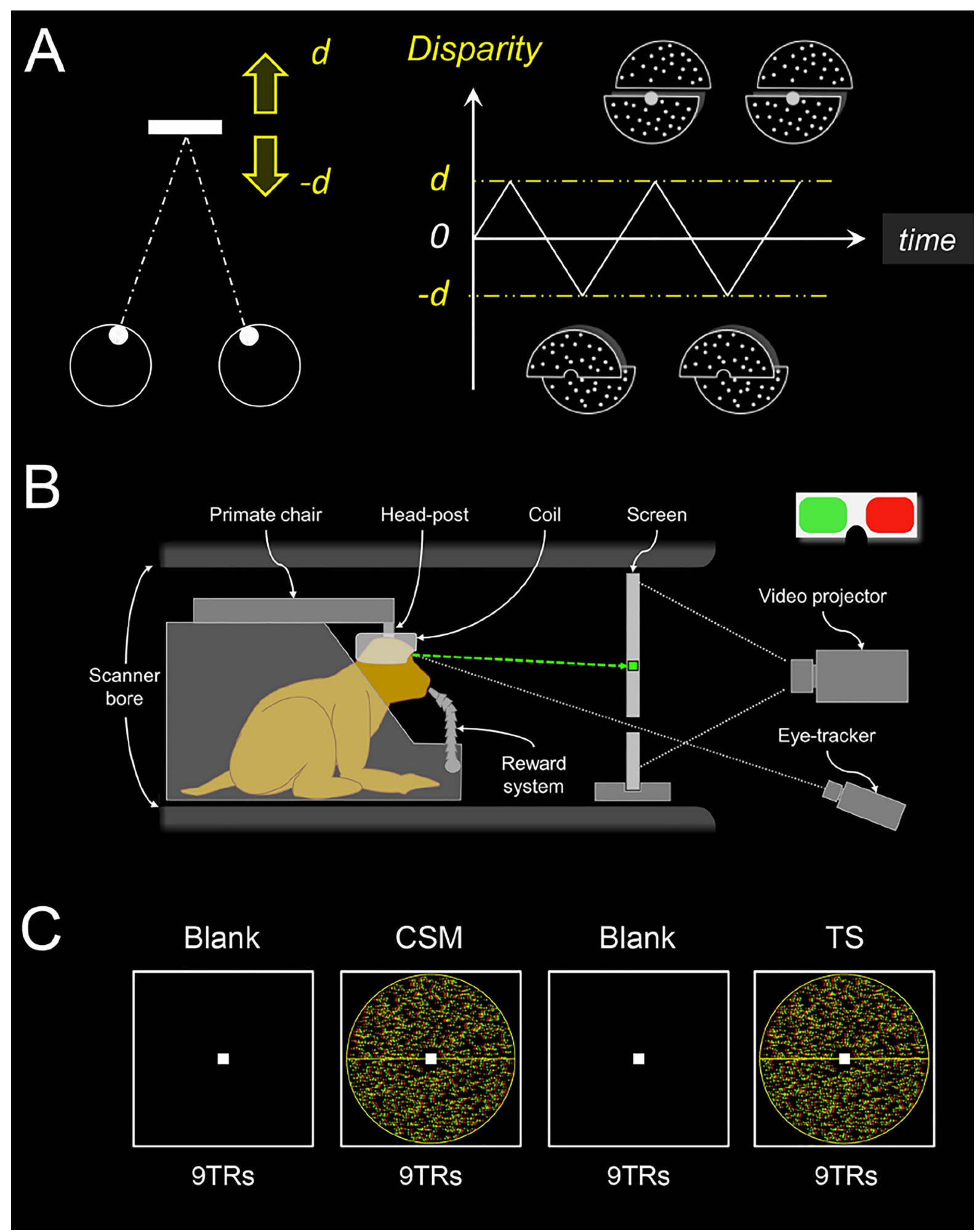

Figure 1. Stimulus design and experimental protocol. (A) In the main condition (CSM), the motion occurs along the antero-posterior axis (leftward panel). The stimulus consisted of a disk (11 ${ }^{\circ}$ of radius) defined by dRDS refreshed at $30 \mathrm{~Hz}$. Its upper and lower parts moved in depth between $d= \pm 23.3$ arcmin in opposition of phase, following a 1-Hz triangular function (rightward panel). In our control condition (TS for temporally scrambled), the individual frames of the CSM condition were shuffled in time to disrupt the smooth change of disparity over time. Our two conditions had identical retinal disparity distributions but only the CSM condition conveyed motion-in-depth. (B) Schematic representation of the monkey fMRI setup. The animal was seated in a sphinx position within the primate chair, in the bore of the scanner, with the 8-channel, phased-array coil placed on top of the head. The animal was involved in a fixation task while its eye position was monitored by an infrared video-based eye-tracker. Horizontal disparity was introduced through red/green anaglyph filters. (C) Illustration of the experimental protocol. Recordings were performed using a blocked design, with the alternation of CSM and TS stimuli separated by blank periods. Each run contained three repetitions of such blocks plus an additional baseline period of 9TRs (117TRs in total). CSM conditions were shown first in half of the runs and TS conditions appeared first in the other half of the runs. 
derived as the response to a 2-s stimulus (our fMRI sampling rate). Note however that our stimulus duration was $4 \mathrm{~s}$ rather than $2 \mathrm{~s}$ because linearity deteriorates at short durations (Boynton et al. 1996; Logothetis and Wandell 2004) and also because this duration was used in a previous monkey fMRI study that characterized the BOLD HRF in macaque (Leite et al. 2002). For each monkey, the average response to the 4-s stimulus was fit as the convolution of the responses to two 2-s responses, each of which is the HRF. We parameterized the HRF as the difference of two gamma functions (Friston et al. 1998). This functional form of the HRF captures the late undershoot of the response better than a single gamma function (Boynton et al. 1996).

General Linear Model and Whole-Brain Univariate Analyses

Univariate statistics were performed at the voxel level in SPM12, using a general linear model (GLM). Our visual (CSM and TS) and baseline conditions were implemented as the three main regressors of the GLM. As reported above, we only analyzed runs with fixation performance greater than $85 \%$. We used the oculometric data of those runs to define regressors of noninterest that were included in the GLM to exclude the possible contribution of eye movements from our analyses. These regressors were obtained by automatically detecting the presence (1) or absence (0) of saccades in the different volumes of every run. The corresponding saccade regressors were then convolved with the HRF and introduced into the model. To characterize and eliminate noise in our recordings, we also performed a principal component analysis on voxels located outside the brain (see Vanduffel and Farivar 2014). Indeed, time courses in those voxels should not be influenced by our experimental design but rather reflect artifacts caused by movements of the animal. For each run, we determined the number of principal components that were necessary to explain $80 \%$ of the variance in these voxels and used the corresponding principal vectors as regressors of noninterest in our model. This adaptive procedure typically added an average of $13.3( \pm 9.3)$ and $11.3( \pm 5.1)$ additional regressors in the models for Monkey 1 (M01) and Monkey 2 (M02), respectively.

We estimated the beta values associated with our GLM using the RobustWLS toolbox (Diedrichsen and Shadmehr 2005), which is provided as an additional toolbox for SPM12 (http://www.diedrichsenlab.org/imaging/robustWLS.html). This approach allows estimating the noise variance for each image in the time series, using the derivative of a maximum likelihood algorithm. Variance parameters are then used to obtain a weighted least square estimate of the regression parameters of the GLM. It therefore helps to reduce the impact of noisier volumes on beta estimation. Previous studies showed that such a method significantly improved estimations in blocked-design fMRI experiments (see Takeuchi et al. 2011). The beta weights obtained from the GLM were subsequently used to perform univariate analyses (t-scores) at the whole brain level. These analyses were performed on the preprocessed EPI data and the beta weights were then projected onto the high-resolution volumes of our two animals. They were also projected on the individual cortical surfaces and on the cortical surface of the F99 template using the Caret software (Van Essen et al. 2001).

Localisation of Areas Selective to Motion-in-Depth and Description of Their Responses

In order to identify areas with specific responses to motionin-depth, we examined the statistical parametric map corresponding to the contrast between our two visual conditions
$(\mathrm{CSM}>\mathrm{TS})$ and thresholded this map at $\mathrm{P}<10^{-3}$ (uncorrected, $t$-value $>3.1$ ). All the cortical regions that showed significantly stronger responses to CSM than to TS in both hemispheres and in each animal were considered. We controlled that these areas overlapped when projected on the right cortical surface of the F99 template. To further document the activations in those areas, we identified their local maxima and considered $3 \times 3 \times 3$ voxel cubes around their coordinates. We then computed the percentage of signal change (PSC) corresponding to our main condition and its control using the following equations:

$$
\mathrm{PSC}_{\mathrm{CSM}}=\left(\beta_{\mathrm{CSM}}-\beta_{\text {baseline }}\right) / \beta_{\text {constant }} \times 100
$$

$$
\mathrm{PSC}_{\mathrm{TS}}=\left(\beta_{\mathrm{TS}}-\beta_{\text {baseline }}\right) / \beta_{\text {constant }} \times 100 .
$$

These values were extracted within small $(3 \times 3 \times 3)$ voxel cubes rather than within patches determined by anatomical and/or statistical criteria, due to the fact that anatomical borders between areas are difficult to determine precisely and that our contrast led to extended activations that cannot be accurately divided into clusters corresponding to different functional regions (see Figs 2-4). Our approach is more conservative and avoids subjectivity when dealing with borders between areas. Importantly, we reproduced our analyses with betas extracted from smaller $(1 \times 1 \times 1)$ or larger $(5 \times 5 \times 5)$ voxel cubes, and this did not impact our results. Note that here we just document activations around the local maxima of selective areas (notably the relative difference between activations in our main condition and in its control and also the variability across runs) but we do not perform additional statistical analyses so as to avoid double dipping (Kriegeskorte et al. 2009).

\section{Definition of Retinotopic Areas and Characterization of Their Responses to Motion-in-Depth}

We also performed a wide-field retinotopic mapping to delineate retinotopic regions that were used for additional ROI-based analyses. Whole-brain images were acquired with an identical setup as for the main experiment. In this case, visual stimuli were displayed using a large field-of-view $\left(80^{\circ}\right.$ of visual angle, viewing distance $=25 \mathrm{~cm}$ ) and consisted of videos of a fruit basket that was moving laterally, forward and backward in monocular viewing. Traditional (clockwise/counter clockwise) rotating wedges (radius: $40^{\circ}$, angular extent: $49^{\circ}$ ) and expanding/contracting rings (eccentricity linearly varying between $0^{\circ}$ and $40^{\circ}$ ) were used as visual apertures through which the fruit basket was displayed. Each run lasted $230 \mathrm{~s}$ and contained five cycles of $44 \mathrm{~s}$ with the first $10 \mathrm{~s}$ of a run being discarded (dummy scans) for the signal to reach its baseline. A small green square $\left(0.4^{\circ} \times 0.4^{\circ}\right)$ at the center of the screen was used to control for fixation during passive viewing. As in our main experiment, only runs with more than $85 \%$ of correct fixation (respectively, 47 and 48 runs for M01 and M02) were kept for further analyses. A preprocessing pipeline similar to the one described above was performed on the selected runs except that no smoothing was applied to the volumes and a fixed number of components (18 components) was used when performing the PCA, later used as a regressor of noninterest in the GLM. We projected the volume data onto individual surfaces using the Caret software (Van Essen et al. 2001) and a custom reorientation algorithm.

A population receptive field (pRF) analysis (Dumoulin and Wandell 2008) was performed using the MATLAB analyzePRF toolbox developed by Kay et al. (2013). For each surface node, an exhaustive set of theoretical pRF parameters (polar angle, 


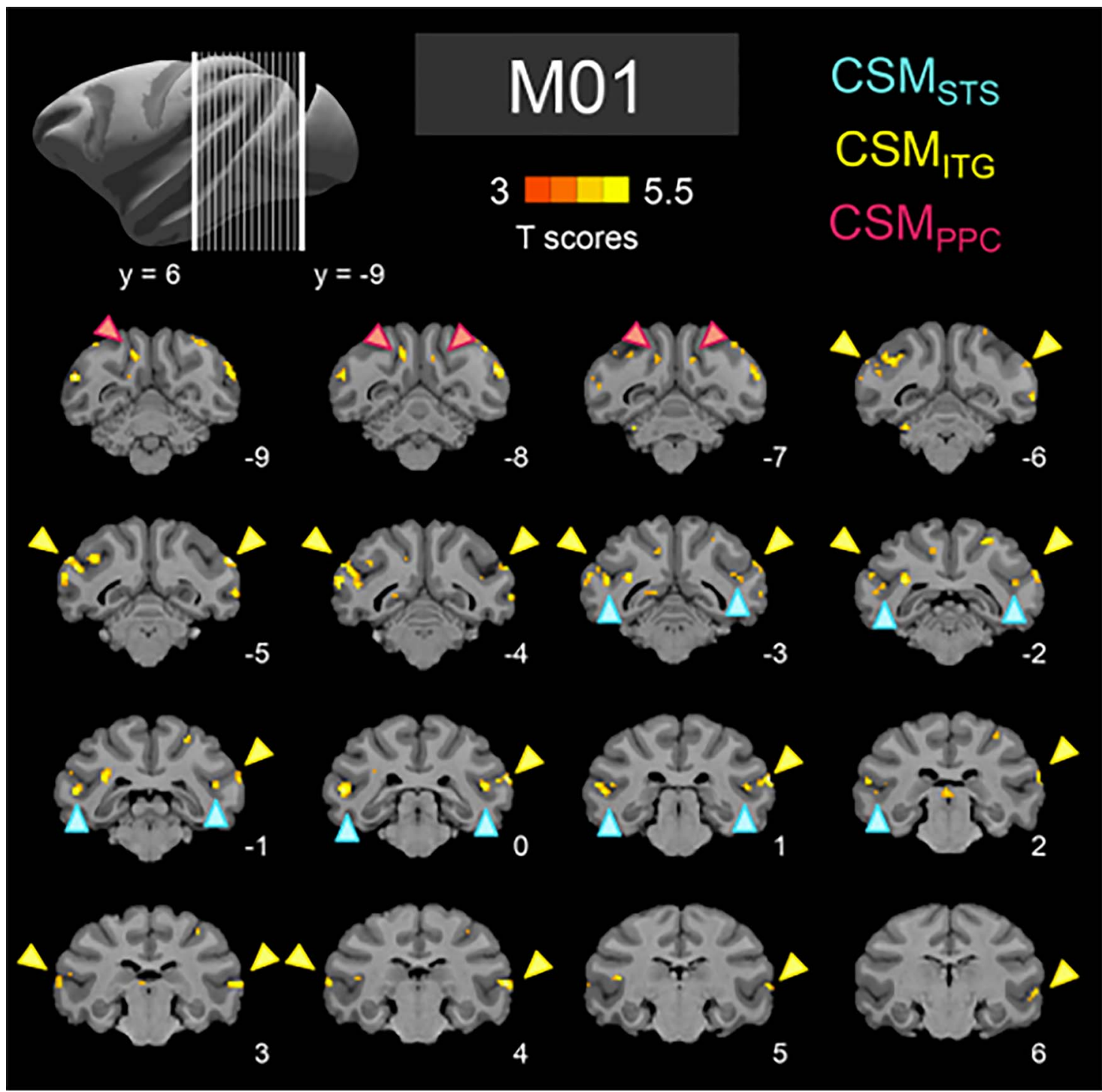

Figure 2. Activations for the contrast between CSM and its temporally scrambled version (TS) for M01. It shows activations that were stronger for the CSM condition than for the TS condition (t-score $>3.1 ; P<10^{-3}$ uncorrected). Data are projected on the individual anatomical template of the macaque and are shown for different

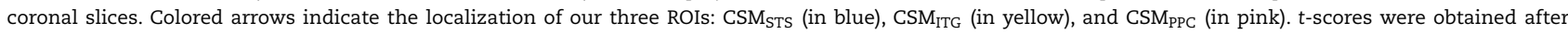
computing the statistical parametric map for the contrast of interest between CSM and TS.

eccentricity, and size) was used to generate time courses that were compared with the real recordings. pRF size and position parameters that best predicted the data were selected as the node pRF parameters. With this approach, we obtained polar angle and eccentricity maps from which we characterized retinotopic areas that were described in previous monkey fMRI studies: V1, V2, V3, V4, V3A, as well as the regions within the STS (V4t, MT, MSTv, and FST) that form the MT cluster as described by Kolster et al. (2009). Those nine retinotopically defined regions were then projected back to the volumetric space to perform a ROI-based analysis of our motion-in-depth data. This was done using the inverse of the transformation between the volumetric and surface spaces mentioned above.

To test whether these retinotopic areas had specific responses to motion in depth, we first estimated their average PSC during the CSM condition and its TS control. We subsequently computed the corresponding difference between
PSCs:

$$
\Delta_{\mathrm{PSC}}=\mathrm{PSC}_{\mathrm{CSM}}-\mathrm{PSC}_{\mathrm{TS}} .
$$

Note that we chose here to use the difference of PSCs because the PSCs for the CSM and TS conditions are paired. In order to estimate whether our observed PSC differences in these retinotopic areas were not due to chance, we computed permutation tests. We randomly attributed a negative sign to our PSC values and computed the mean difference, repeating this procedure 10000 times. We then calculated a P-value defined as the proportion of random differences that were superior to our observed difference.

\section{D Motion Localisers}

To determine whether the regions that have specific responses to binocular 3D motion are also responsive to 2D motion, we performed a control experiment in which we contrasted responses 


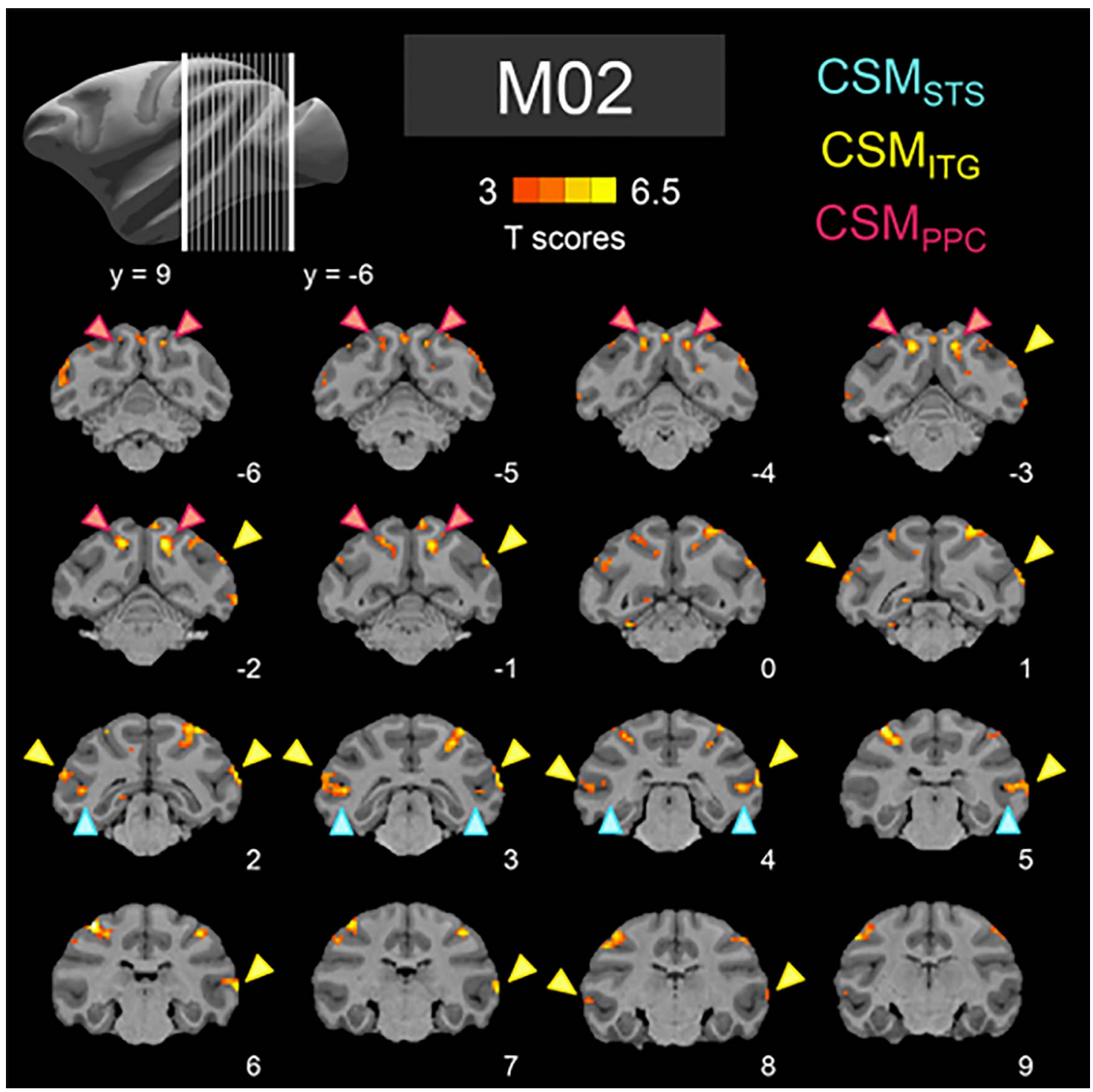

Figure 3. Activations for the contrast between CSM and its temporally scrambled version (TS) for M02. Conventions are similar to Figure 2.

to static images versus rich $2 \mathrm{D}$ motion stimuli. The scanning procedure was identical to the main experiment procedure. Motion localizer stimuli were based on the fruit basket video used for the retinotopic mapping experiment. For the static version, static images were randomly drawn from the video and refreshed at $1 \mathrm{~Hz}$. For the moving version, the video was normally played. Stimuli were displayed either centrally $\left(<3^{\circ}\right.$ of eccentricity) or peripherally ( $>3^{\circ}$ of eccentricity). As for the retinotopic experiment (see the previous section), these visual stimuli were displayed using a large field-of-view $\left(80^{\circ}\right.$ of visual angle) at a viewing distance of $25 \mathrm{~cm}$. Each visual condition lasted $6 \mathrm{~s}$ and was interleaved with a 10-s baseline. The four visual conditions were presented in a pseudo-randomized order and were repeated three times within each run. Five extra baseline scans were added at the beginning of a trial for the signal to reach its baseline, thus resulting in a total duration of $202 \mathrm{~s} \mathrm{(101}$ TRs) for each run. In total 42 and 26 runs with fixation above $85 \%$ were kept for our analyses. Selected data was preprocessed as previously described, with an adaptive number of components that were necessary to explain $80 \%$ of the variance for each run, adding an average of $12.6( \pm 10.1)$ and $11.9( \pm 3.7)$ additional regressors in the model.

To estimate motion sensitivity in our ROIs and in our retinotopic areas, we contrasted moving and static conditions, by combining central and peripheral presentations. We then performed a ROI-based analysis, looking at the BOLD activity within our independently defined regions.

\section{Results}

The aim of this study was to identify the cortical network that processes disparity-defined motion-in-depth (i.e., CSM) in two awake, behaving macaques using functional magnetic resonance imaging. Our experimental design was directly derived from previous human neuroimaging studies (Likova and Tyler 2007; Rokers et al. 2009; Kaestner et al. 2019) so as to determine the homologies but also the differences between the BOLD activations in the two species (Orban 2002). Our 

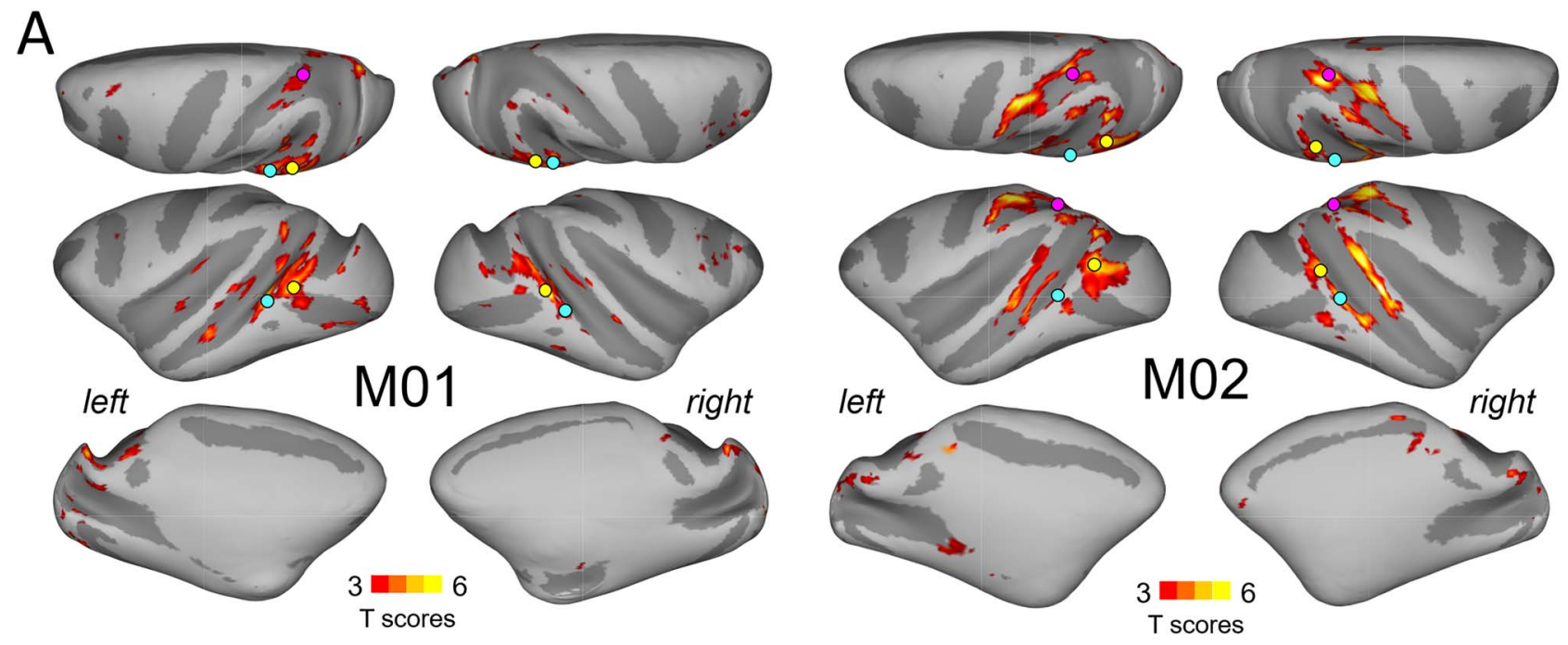

B
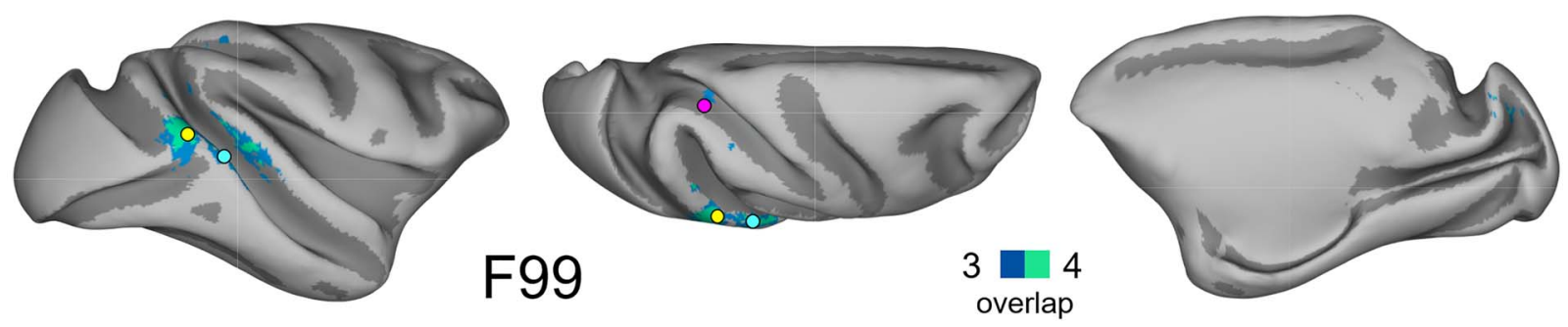

C

M01 M02
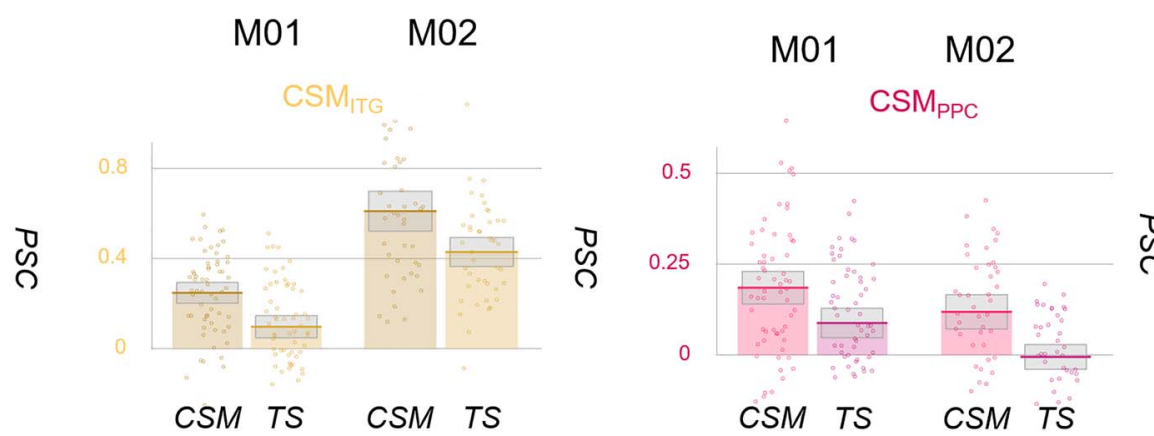

Figure 4. Activations for the contrast between CSM and its temporally scrambled version (TS) projected onto individual cortical surfaces and on the F99 template. (A) Activations that were stronger for the CSM condition than for the TS condition. Data were thresholded at $P<10^{-3}$ (uncorrected) and projected on the individual cortical surfaces of each animal (dorsal, frontal, and medial views). Colored dots indicate the localisation of our three ROIs: CSMSTS (in blue), CSM $\mathrm{ITG}_{\text {(in }}$ yellow), and CSMPPC (in pink). (B) Degree of overlap between the activations found in the two hemispheres of the two animals. The four individual cortical surfaces were morphed onto the right cortical surface of the F99 macaque template for projection of all the thresholded maps (frontal, dorsal and medial views). Blue color indicates the overlap of $3 / 4$ hemispheres and green color of $4 / 4$ hemispheres. (C) PSCs for the two visual conditions (CSM and TS) with respect to baseline (fixation on a black screen) in our three ROIs. The boxes give the $95 \%$ confidence intervals for the average values. The dots provide the data for each run. A small jitter was introduced to facilitate visibility.

CSM condition and its temporal scramble (TS) control were defined from dynamic random-dot stereograms (dRDS). They had identical retinal disparity distributions but differ in their temporal sequences (see Materials and Methods). Only the CSM condition conveyed motion-in-depth. Figures 2 and 3 show the statistical parametric maps obtained for the contrast between CSM and TS on the individual anatomical templates of each subject (M01 on Fig. 2 and M02 on Fig. 3). These data are shown for different coronal slices.

On these two figures, red-to-orange colors indicate significantly stronger BOLD activations for the CSM condition than for the TS condition $\left(P<10^{-3}\right.$, uncorrected). Despite differences in the activation patterns observed in the two animals, this analysis reveals a network encompassing the temporal and parietal cortices in both monkeys. Notably, three cortical areas are consistently found in both the left and the right hemispheres of our two macaques. Colored arrows show these areas. For the sake of comparison with previous human neuroimaging studies, we named those areas after Likova and Tyler's denomination (Likova and Tyler 2007), that is, CSM for cyclopean stereomotion responsive areas. The first area $\left(\mathrm{CSM}_{\mathrm{STS}}\right)$ is located on the posterior bank of the STS and extends posteriorly on the 
Table 1 MNI coordinates (in $\mathrm{mm}$ ) of the local maxima for the 3 regions that were significantly more responsive for the CSM condition than for the TS control in the two hemispheres of the two animals

\begin{tabular}{|c|c|c|c|c|c|c|}
\hline \multirow[t]{2}{*}{ ROI } & \multicolumn{3}{|c|}{ M01 } & \multicolumn{3}{|c|}{ M02 } \\
\hline & $\mathrm{x}$ & $\mathrm{y}$ & z & $\mathrm{x}$ & $\mathrm{y}$ & $z$ \\
\hline \multicolumn{7}{|c|}{$\mathrm{CSM}_{\mathrm{STS}}$} \\
\hline $\mathrm{L}$ & -23 & 0 & 15 & -19 & 3 & 16 \\
\hline $\mathrm{R}$ & 21 & 0 & 17 & 20 & 4 & 16 \\
\hline \multicolumn{7}{|c|}{$\mathrm{CSM}_{\mathrm{ITG}}$} \\
\hline $\mathrm{L}$ & -29 & 4 & 15 & -23 & 3 & 18 \\
\hline $\mathrm{R}$ & 28 & 1 & 20 & 26 & 3 & 19 \\
\hline \multicolumn{7}{|c|}{$\mathrm{CSM}_{\mathrm{PPC}}$} \\
\hline $\mathrm{L}$ & -5 & -8 & 28 & -6 & -3 & 30 \\
\hline $\mathrm{R}$ & 5 & -7 & 27 & 6 & -2 & 30 \\
\hline
\end{tabular}

infero-temporal gyrus. The second one $\left(\mathrm{CSM}_{\mathrm{ITG}}\right)$ is located on the infero-temporal gyrus, at the intersection between the lunate sulcus, the inferior occipital sulcus (IOS) and the STS. The last area $\left(\mathrm{CSM}_{\mathrm{PPC}}\right)$ is localized in the posterior parietal cortex (PPC), mostly on the medial bank of the IPS (even though activations can also be observed on its lateral bank in M02). This area might therefore correspond to the posterior intraparietal (PIP) area. To be sure that the anatomical localizations of these three areas are not affected by our projections on the individual anatomical $\left(T_{1}\right)$ images, we confirmed their position on the functional (EPI) images in both monkeys (see Supplementary Fig. S1). The MNI coordinates corresponding to the local maxima of these areas in the two animals are provided in Table 1.

To demonstrate the consistency of these results across hemispheres, we show in Figure 4 the projections of these activations on the individual cortical surfaces (see panel A).

As can be observed, our three ROIs are found in all the individual surfaces, even though $\mathrm{CSM}_{\mathrm{PPC}}$ is less visible in the right hemisphere of M01. This was confirmed by our projections of these activations on the right hemisphere of the F99 template. Figure $4 \mathrm{~B}$ shows that our three regions overlap in at least three hemispheres for CSM $_{\mathrm{PPC}}$ and in four hemispheres for $\mathrm{CSM}_{\mathrm{STS}}$ and $\mathrm{CSM}_{\mathrm{ITG}}$. The bar graphs in Figure $4 \mathrm{C}$ provide the activations elicited by our CSM condition and its TS control relative to baseline (blank screen) around those local maxima (see Material and Methods). The thick lines provide the average values, and the boxes give the corresponding $95 \%$ confidence intervals.

Finally, it is worth noting that in monkey M02, significant BOLD activations were also found in more anterior parts of the IPS notably within the ventral and anterior intraparietal areas (VIP and AIP, respectively). VIP has been shown to be involved in egomotion-compatible optic flow processing in both monkey (Cottereau et al. 2017) and human (Wall and Smith 2008), whereas AIP has been suggested to play a role in 3D object processing and visually guided hand movements in both species as well (Sakata et al. 1997; Durand et al. 2007; Shikata et al. 2007). However, these activations were not confirmed in the second animal, potentially revealing a greater intersubject variability in higher order areas. In M02, activations were also found on the anterior part of the STS but they reflect responses from the fundus of the STS and/or from its posterior bank that were smoothed by our preprocessing pipeline and/or our transformations from the volume to the individual surfaces. The activations observed in the anterior part of the STS actually belong to clusters centered on the posterior bank.

Finally, it is important to emphasize here that we did not observe significant CSM responses on the cortex medial faces.
Neither the anterior bank of the parieto-occipital sulcus, where the motion sensitive area V6 is located (see Pitzalis et al. 2013), nor the posterior part of the cingulate sulcus, where our group previously identified an area (pmCSv) responsive to egomotioncompatible optic flow (Cottereau et al. 2017), seems to have strong sensitivity to motion-in-depth.

\section{Retinotopic Analysis}

Previous studies in human found that the hMT+ complex had significant responses to stereomotion, notably based on changing disparity over time (CDOT) (Rokers et al. 2009; Joo et al. 2016). A single-cell study in macaque also found a weak but significant selectivity to CDOT in area MT (Sanada and DeAngelis 2014). In order to determine whether the CSM-responsive ROIs we obtained from our univariate analyses overlap with (or correspond to) area MT and/or its neighbor regions, we performed a retinotopic mapping in our two animals (see more details in Materials and Methods). This allowed us to delineate the areas of the MT cluster: V4t, MT, MSTv, and FST (see Kolster et al. 2009), which is not possible with more classical motion localizers of the MT/hMT+ complex (even though some human studies proposed solutions to separate hMT from hMST, see Huk et al. 2002). In Figures $5 \mathrm{~A}$ and $6 \mathrm{~A}$, we show the locations of these areas and of our two CSM-responsive regions around the STS and the ITG, $\mathrm{CSM}_{\mathrm{STS}}$ and $\mathrm{CSM}_{\mathrm{ITG}}$, respectively. Figures $5 B$ and $6 \mathrm{~B}$ present the activations for the contrast between CSM and its temporally scrambled version (TS) projected on the same views.

We can see that although our two regions are close to the MT cluster, they do not overlap with it. Area CSM STS $_{\text {is located }}$ more anteriorly along the posterior bank of the STS. Area CSM $_{\text {ITG }}$ is located more posteriorly on the ITG, in a position that might correspond to areas V4 and/or V4A (see Discussion).

To complete our study and to allow a direct comparison with previous human data (Likova and Tyler 2007; Rokers et al. 2009; Kaestner et al. 2019), we performed ROI-based analyses within retinotopically defined areas constituting the MT cluster as well as the early-to-intermediate visual cortex: V1, V2, V3, V4, and V3A. The differences between the percentages of signal change ( $\triangle \mathrm{PSC}$ ) for CSM versus TS in our two macaques are shown in Figures $5 \mathrm{~B}$ and $6 \mathrm{~B}$ for visual areas (V1, V2, V3, V3A, and V4) and for the MT cluster (V4t, MT, MSTV, and FST). We can observe that if CSM selectivity in all these areas is not as pronounced as in $\mathrm{CSM}_{\mathrm{STS}}, \mathrm{CSM}_{\mathrm{ITG}}$, and $\mathrm{CSM}_{\mathrm{PPC}}$, responses in the MT cluster tend to be stronger than those measured in V1, V2, and V3. Permutation tests demonstrated significant CSM effects in areas MT and V4t (1/4 hemispheres), MST (2/4 hemispheres, right hemispheres 

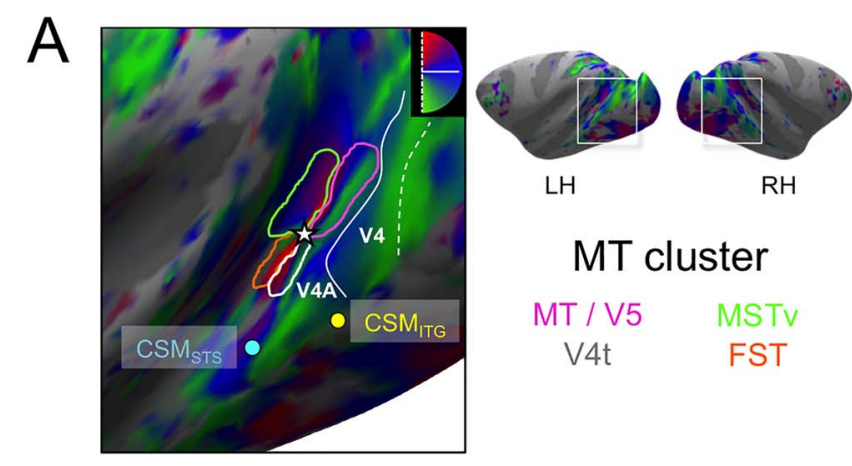

\section{MT cluster}

MT / V5 MSTV

V4t FST

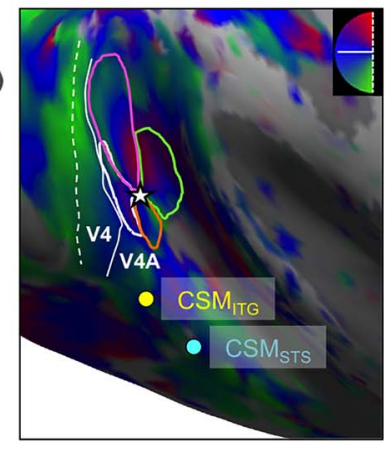

B
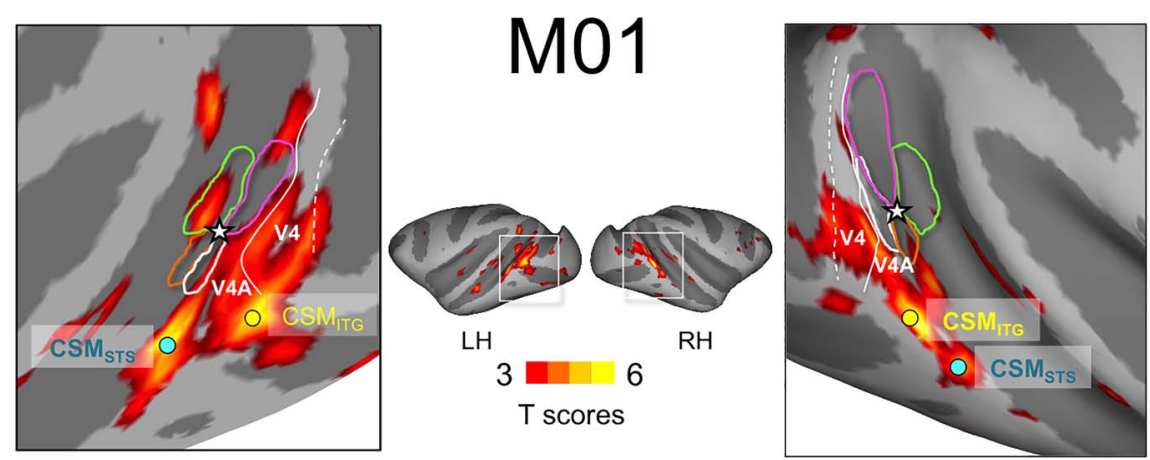

MT cluster
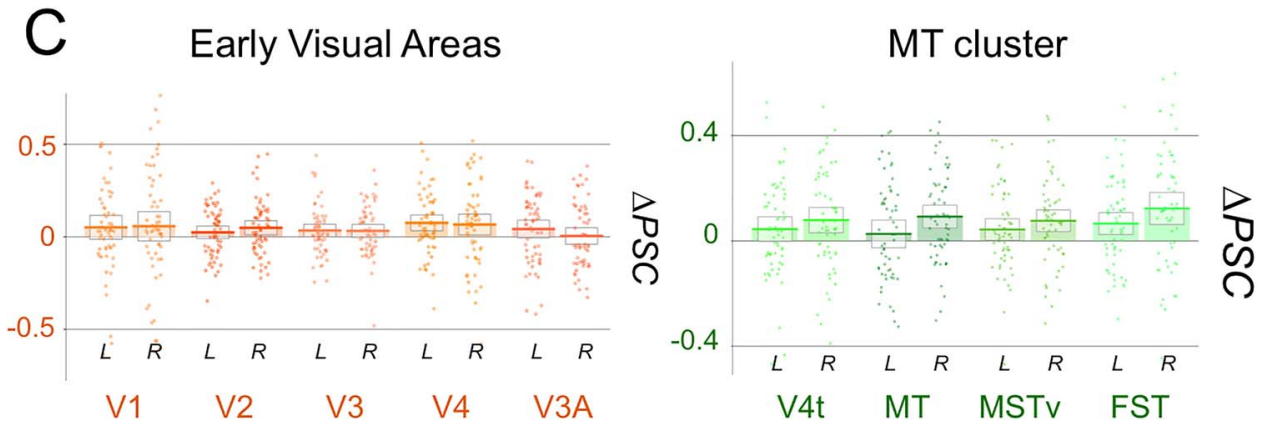

Figure 5. (A) Retinotopic mapping of the STS for M01 and delimitation of the MT cluster areas: MT (dark blue), V4t (pink), MSTv (orange), and FST (green). We show here the polar angle maps that were used to delineate the borders between these areas. The extent of those areas was obtained from the eccentricity maps. We also show the representations of the vertical and horizontal meridians that delineate the borders between V3 and V4 and between V4 and V4A. Colored dots indicate the local maxima positions for areas CSMSTS (in blue) and CSM $_{\text {ITG }}$ (in yellow). As shown on the maps, CSMSTS and CSM ITG are in the vicinity of the MT cluster, but clearly exterior to it. (B) Activations for the contrast between CSM and its temporally scrambled version (TS) projected on the individual surfaces of M01, for both left and right hemispheres. (C) Difference in percentages of signal change ( $\triangle \mathrm{PSC}$ ) between the CSM and TS conditions in retinotopic areas. On the left, thick lines of the bar graphs provide average values for the left and right hemispheres of visual areas: V1, V2, V3, V4, and V3A. On the right, average values are given for the MT cluster areas: V4t, MT, MSTv, and FST. The boxes give the $95 \%$ confidence intervals for the average values. The dots provide the data for each run. A small jitter was introduced to facilitate visibility.

only), and FST ( $3 / 4$ hemispheres). This suggests the presence of a marginal selectivity to CSM in these regions. We also found that responses were significantly stronger for motion in depth in area V4 for one animal (two hemispheres) but not for the other. Responses to CSM were not significantly stronger in V3A.

\section{D Motion Analysis}

To test whether our three regions are only responsive to motionin-depth or whether they are activated by motion in general, and notably by 2D motion, we ran an additional motion localizer in our two animals ( $n=42$ and 26 runs in M01 and M02, respectively, see more details in Materials and Methods). We then computed the difference between the percentages of signal change $(\triangle \mathrm{PSC})$ corresponding to the $2 \mathrm{D}$ motion and static image conditions. As expected from such a localizer, this analysis led to significantly stronger responses to motion in most of the retinotopic areas and more specifically within areas of the MT cluster. In particular, permutation tests demonstrated that all four regions of the MT cluster had significantly stronger responses to $2 D$ motion in the two animals $(P<0.05$, except for left V4t in M02). We show in Figure 7 the results of these analyses in our three CSM-responsive areas $\left(\mathrm{CSM}_{\mathrm{STS}}, \mathrm{CSM}_{\mathrm{ITG}}\right.$, and $\mathrm{CSM}_{\mathrm{PPC}}$ ).

We can observe that only $\mathrm{CSM}_{\mathrm{STS}}$ and $\mathrm{CSM}_{\mathrm{ITG}}$ have a significant response to 2D motion (permutation tests, $P<0.05$ ), in both hemispheres for $\mathrm{CSM}_{\mathrm{STS}}$ and in the left hemisphere for $\mathrm{CSM}_{\text {ITG }}$, for each monkey. Their motion selectivity (in particular in $\mathrm{CSM}_{\mathrm{STS}}$ ) is therefore not specific to CSM. On the opposite, responses to $2 \mathrm{D}$ motion in area $\mathrm{CSM}_{\mathrm{PPC}}$ are not different 

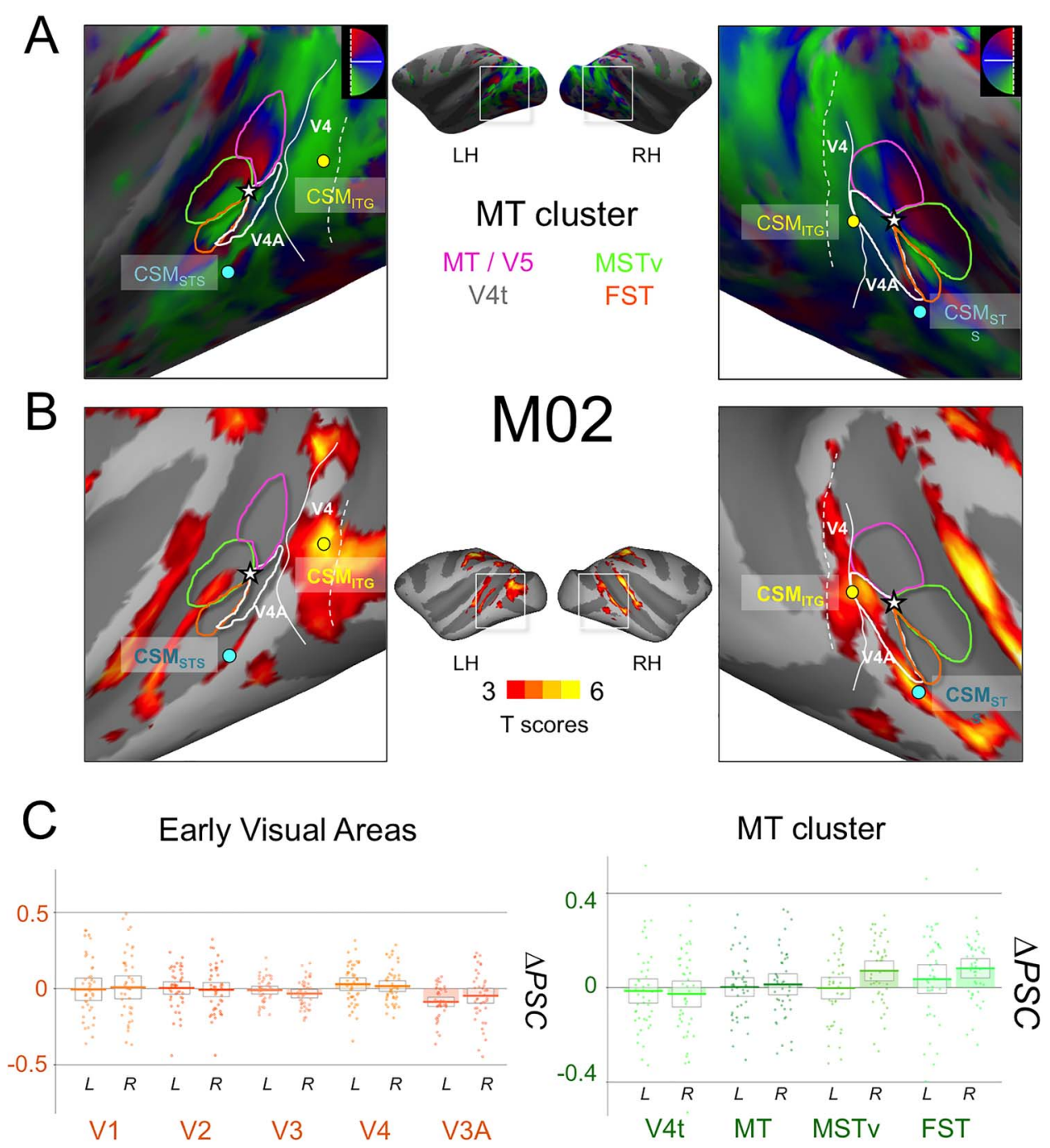

Figure 6. (A) Retinotopic mapping of the STS for M02 and delimitation of the MT cluster areas: MT (dark blue), V4t (pink), MSTv (orange), and FST (green). (B) Activations for the contrast between CSM and its temporally scrambled version (TS) projected on the individual surfaces of M02, for both left and right hemispheres. (C) Difference in percentages of signal change ( $\triangle \mathrm{PSC}$ ) between the CSM and TS conditions in retinotopic areas. Conventions are similar to Figure 5.

from responses to static patterns (permutation tests, $P>0.1$ ). It implies that this region might uniquely respond to CSM.

\section{Selectivity to 3D versus 2D Motion within the Lower Bank of the STS}

To further characterize the selectivity to $3 \mathrm{D}$ and 2D motion within the STS, we defined a path running along the posterior bank of this sulcus on the cortical surfaces of each hemisphere of our two animals. Each path departs from MT area and ends

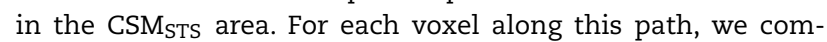
puted the average t-score within its first order neighborhood (i.e., within a $3 \times 3 \times 3$ cube centered on this voxel) for both the stereomotion versus temporal scramble and 2D motion versus static images contrasts. As t-scores for the second contrast were usually higher, we normalized the values along each path by dividing them by the maximum t-scores along the path. This facilitates comparisons between the sensitivity profiles for $3 \mathrm{D}$ and 2D motion. As shown in Figure 8A, these profiles suggest that selectivity to stereomotion varies within the STS, with lower t-score values within the MT cluster and higher values in the $\mathrm{CSM}_{\mathrm{STS}}$ area. By contrast, both CSM areas (CSM $\mathrm{CTS}_{\mathrm{TS}}$ and $\left.\mathrm{CSM}_{\mathrm{ITG}}\right)$ and the MT cluster exhibit strong sensitivity to 2D motion. This observation was confirmed by computing the average percentages of signal changes ( $\triangle \mathrm{PSC}$ ) for $2 \mathrm{D}$ and $3 \mathrm{D}$ motion in those two regions (N.B.: the value for the MT cluster is the average $\triangle \mathrm{PSC}$ across the path). The corresponding bar graphs are shown in Figure 8B.

\section{Discussion}

The aim of the present study was to characterize the cortical areas that process CSM in rhesus macaque. To that end, we adapted the experimental protocols of previous human fMRI studies that investigated the cortical processing of motion in depth (Likova and Tyler 2007; Rokers et al. 2009; Kaestner et al. 2019). Our main condition (CSM) and its control (TS for temporally scrambled) shared the same disparity distribution and were monocularly identical (Fig. 1) but only the CSM condition conveyed stereomotion, since the temporal sequence was 


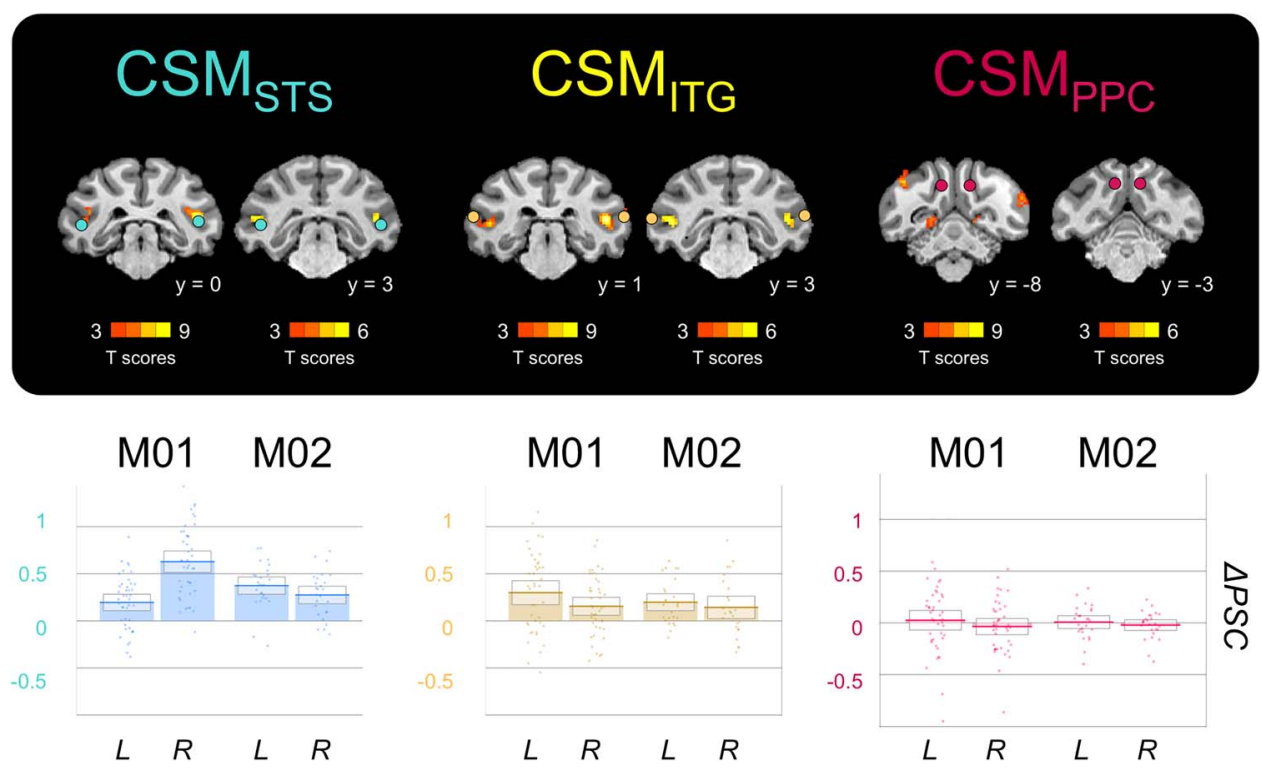

Figure 7. Sensitivity to 2D motion in CSMSTs, $\mathrm{CSM}_{\mathrm{ITG}}$, and $\mathrm{CSM}_{\mathrm{PPC}}$. Stronger responses to 2D motion than to static snapshots of the same video sequences are shown on coronal slices from the individual anatomical template of each animal (upper panel). The color dots provide the position of CSM $\mathrm{STS}_{\mathrm{ST}}$, $\mathrm{CSM}_{\mathrm{TTG}}$, and CSM $\mathrm{PPC}_{\mathrm{PPC}}$. For these three regions, PSC difference between responses evoked by 2D motion and static images is shown on the lower panel. The thick lines of the bar graphs provide the average values across runs for the left (L) and right (R) hemispheres of the two monkeys (M01 and M02). The boxes give the $95 \%$ confidence intervals for these average values. The dots provide the data for each run. A small jitter was introduced to facilitate visibility.

A

$\mathrm{B}$
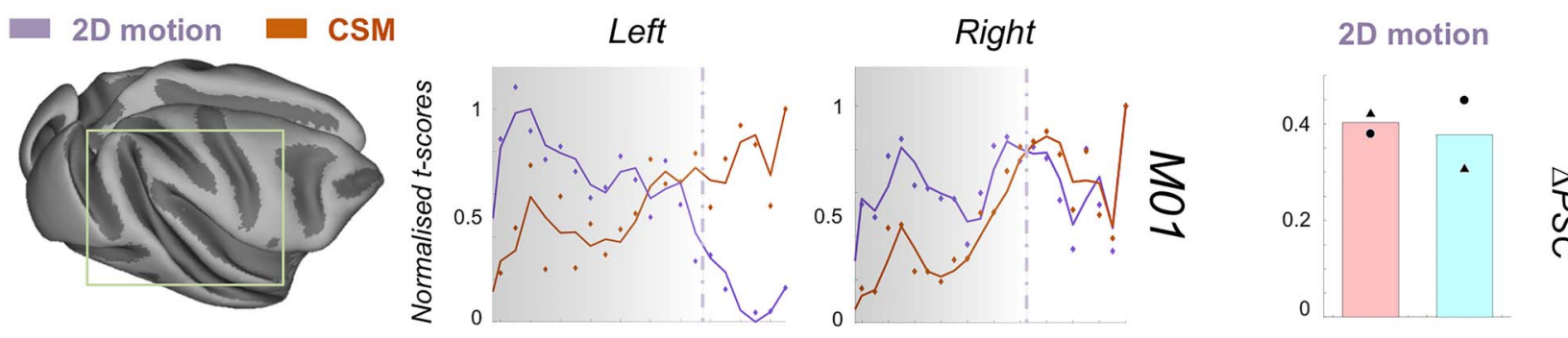

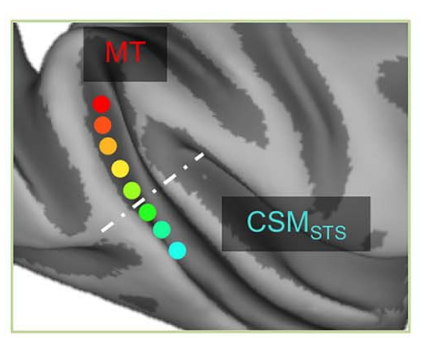

Path along the STS
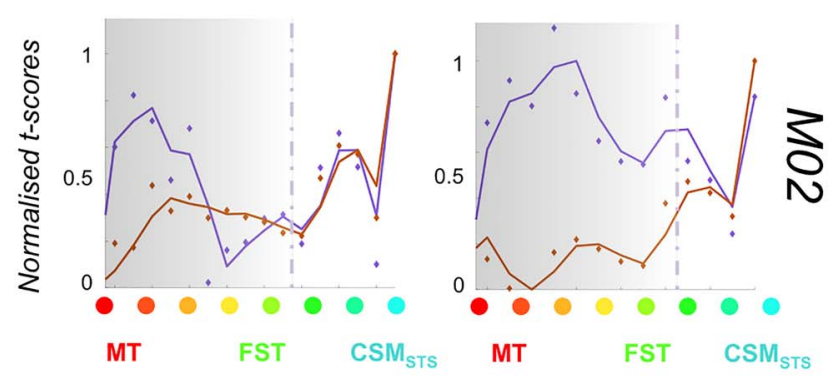

MT

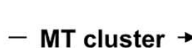

$\mathrm{CSM}_{\mathrm{STS}}$

- MT cluster $\rightarrow$
- MT cluster $\rightarrow$

Figure 8. Selectivity to CSM and 2D motion within the STS. (A) The left panel is a schematized view of the path drawn along the lower bank of the STS, starting from MT area (red dot) and ending within the CSM $\mathrm{STS}_{\mathrm{S}}$ area (cyan dot) defined from the stereomotion versus temporal scramble contrast. The gray dotted line represents the end of the MT cluster. On the right panel, responses to the cyclopean stereomotion (CSM, i.e., stereomotion versus its temporal scramble control) and 2D motion (i.e., 2D motion versus static images) contrasts are respectively shown in orange and purple for each hemisphere of both macaque subjects. Dots provide the normalized $t$-score values along the path whilst the curves were obtained from a median filtering of these values. The general trend is an increase of CSM selectivity along the STS, with the highest value outside the MT cluster. Selectivity to 2D motion peaks in the MT cluster and tends to decrease along the STS path. (B) Average differences in percentages of signal change ( $\triangle \mathrm{PSC}$ ) values in response to both types of tested motions (2D and CSM) in the four MT cluster areas (red bars) and in the CSM $\mathrm{STS}_{\text {area }}$ (cyan bars) for both subjects (triangle symbols for M01 and circle symbols for M02). 
scrambled in the TS condition. We recorded whole-brain BOLD responses from two behaving macaques involved in a passive fixation task using a blocked design. Our analyses revealed a network of three areas whose responses to our CSM condition were consistently (i.e., across hemispheres and animals) stronger than those to our control condition (Figs 2-4). In reference to the original study of Likova and Tyler (2007), we labeled those regions $\mathrm{CSM}_{\mathrm{STS}}, \mathrm{CSM}_{\mathrm{ITG}}$, and $\mathrm{CSM}_{\mathrm{PPC}}$. To complete these analyses, we also documented responses to our CSM condition in visual areas estimated from independent wide-field retinotopic mapping procedures (Figs 5 and 6).

Our $\mathrm{CSM}_{\mathrm{STS}}$ region is located on the inferior bank of the STS and extends on the infero-temporal gyrus (see Figs 2-4), at a location (see Table 1) anterior to the MT area and its satellites (V4t, MSTv, and FST, see Figs 5 and 6). Based on our retinotopic maps, we confirmed that this region is outside the MT cluster (only a marginal overlap with area FST was found in the right hemisphere of M02). Our additional motion localizer demonstrated that it is also responsive to 2D motion (Fig. 7). Previous studies in macaque reported additional motion-sensitive regions in anterior portions of the STS. Using fMRI, Nelissen et al. (2006) notably documented an area in the lower superior temporal sulcus that responds to opponent motions and to actions. This area was 6-8 $\mathrm{mm}$ anterior to FST and therefore does not fully coincide with our $\mathrm{CSM}_{\mathrm{STS}}$ area. Another monkey fMRI study found several regions of the macaque inferior temporal cortex that had specific responses to disparity-defined stimuli (Verhoef et al. 2015). Among them, a region labeled "Pd" (for posterior disparity) was localized in the lower bank of the STS, at a position that matches very well with our CSM $_{\text {STS }}$ area. CSM STS might thus be a distinct motion- and disparity-selective area of the STS that notably processes motion in depth. In human, two studies (Likova and Tyler 2007; Kaestner et al. 2019) found specific responses to CSM in a cortical region anterior to the hMT+ cluster: CSM (N.B.: Rokers et al. (2009) only performed ROIbased analyses and it is therefore not possible to know whether they also had significant responses to motion in depth in this region). Our $\mathrm{CSM}_{\mathrm{STS}}$ area might therefore be its macaque homologue. It is nonetheless important to note here that in the human studies, the delineation of the hMT + complex (and sometimes its hMT and hMST subregions) was based on a contrast between the responses to uniform versus random motion whereas in our case the MT cluster was obtained from retinotopic mapping. To further clarify the potential homology between human CSM and macaque $\mathrm{CSM}_{\mathrm{STS}}$, it would be interesting for future human studies to properly define area MT and its satellites using retinotopic mapping (see Kolster et al. 2010) in order to precisely determine the location of the CSM area with respect to those regions.

Our stereomotion contrast was based on a smooth variation in depth versus its temporally scrambled version (as in Kaestner et al. (2019) and in the TS control of the second experiment of Rokers et al. (2009)). Although we used dRDS, it is possible that this temporally scrambled control still evokes some apparent percept of motion. However, it lacked the smooth change of disparity of our main condition. In their experiments, Likova and Tyler (2007) used two planes that alternated between two different depths (thereby generating an apparent motion in depth) in their main condition whereas their corresponding control was a plane at a unique depth. We hypothesize that in both human and

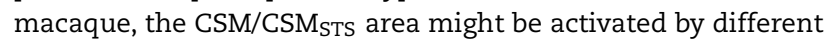
types of motion in depth, and notably by our contrast and the one used by Likova and Tyler (2007). This hypothesis is further supported by a control performed by these authors (see their supplementary materials) that demonstrated that significant activations were also obtained in this area with stimuli smoothly varying in depth (i.e., where binocular disparity was changed according to a sine wave), being therefore closer to those used in our own study.

Although our univariate statistics did not show significant responses in the MT area and its satellites, ROI-based analyses demonstrated that for some animals and/or hemispheres, responses in V4t (1/4 hemispheres), MT ( $1 / 4$ hemisphere), MSTv (2/4 hemispheres), and FST (3/4 hemispheres) were significantly stronger for our stereomotion condition (Figs 5 and 6). In a pioneer study, Maunsell and Van Essen (1983) concluded from single-cell recordings in area MT of anesthetized macaques that neurons in this region had no selectivity to motion-in-depth (see also Felleman and Kaas 1984). More recently, Sanada and DeAngelis (2014) found, using a different method, that MT does host neurons tuned to motion-in-depth (see also Czuba et al. 2014) but that these neurons were mostly driven by the IOVD between the two eyes with only a modest contribution of the change of disparity over time (CDOT): $10 \%$ of their neurons had significant selectivity for CDOT versus $\sim 57 \%$ for IOVD. These findings are in line with our study and suggest that if selectivity to stereomotion is observable in area MT, it remains moderate. To our knowledge, selectivity to motion-in-depth was not directly tested in areas MSTv and FST. Based on our data, we hypothesize that a larger proportion of neurons tuned to CSM could be found there. Altogether, the responses we measured in the STS are consistent with a model where stereomotion would be progressively integrated along a posterior-to-anterior axis with moderate responses in MT, intermediate responses in areas MSTV and FST, and stronger responses in CSM $\mathrm{CTS}_{\text {. This }}$ hypothesis is supported by our analysis of the responses on a path defined along the STS (see Fig. 8), which suggests that selectivity to stereomotion progresses beyond area MT.

Using an ROI-based analysis, all three previous human studies found significant responses to motion in depth in the hMT+ cluster. Likova and Tyler (2007) reported that selectivity in this cluster was weaker than in their CSM region, in agreement with what we found in macaque. In contrast, Kaestner et al. (2019) found that responses in hMT+ (in both hMT and hMST) were stronger than in CSM (see their Fig. 7). Given their use of a relatively small field of view (i.e., their stimuli had $5^{\circ}$ of radius) contrasting with much larger stimuli in our experimental proto$\mathrm{col}\left(11^{\circ}\right.$ of radius) and in Likova and Tyler's experiment (i.e., their display was a square of $30 \times 40^{\circ}$ ), one possibility would be that neurons in the $\mathrm{CSM}_{\mathrm{STS}}$ and CSM regions prefer more eccentric $\left(>5^{\circ}\right)$ stimuli. We computed the average pRF eccentricities and

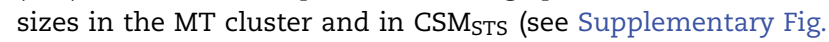
$\mathrm{S} 3 \mathrm{~B}, \mathrm{D}$ ) and showed that in $\mathrm{CSM}_{\mathrm{STS}}$ these parameters are actually similar to those found in V4t and FST, thus discarding this hypothesis. Further studies, notably in human where retinotopic mapping could be used to better define the position of CSM with respect to area MT and its satellites, will be needed to clarify this point.

Our $\mathrm{CSM}_{\text {ITG }}$ region is located on the infero-temporal gyrus, at the intersection of the lunate sulcus, the IOS, and the STS (see Figs 2-4). It is therefore posterior to the MT cluster (see Figs 5 and 6). This location matches well with area V4A that was previously described using single-cell recordings (Pigarev et al. 2002) and fMRI (Kolster et al. 2014). CSM ITG $_{\text {also overlaps }}$ with area V4, as suggested by our retinotopic analyses for which responses to motion in depth were significantly stronger in this area for one animal (see also panel B of Figs 5 and 6). 
Unfortunately, SNRs in our retinotopic data were not sufficient to properly map area V4A (notably its anterior border with area OTd) and further studies will be needed to clarify its responses to stereomotion relatively to those estimated in V4. Both V4 and V4A are activated by disparity (Watanabe et al. 2002; Verhoef et al. 2015) and motion (Li et al. 2013; Kolster et al. 2014), even though their motion selectivity is not as pronounced as in the MT cluster (Kolster et al. 2014). This in line with our finding that $\mathrm{CSM}_{\mathrm{ITG}}$ has only moderate responses to $2 \mathrm{D}$ motion, notably when compared with motion responses in area CSM $_{\text {STS }}$ (Fig.7). Interestingly, two human studies on motion in depth (Likova and Tyler 2007; Kaestner et al. 2019) reported significant CSM responses in area V4. Kaestner et al. (2019) also found strong steremotion responses in area LO-1 (and Rokers et al. (2009) in area LO which includes LO-1) which was proposed to be the human homologue of area V4A (Kolster et al. 2014). These results suggest a good correspondence between CSM responses in those regions of the human and macaque brains.

Area $\mathrm{CSM}_{\mathrm{PPC}}$ is localized in the PPC, mostly on the medial bank of the IPS. Responses in this region were not stronger for 2D motion than for static stimuli, in agreement with previous monkey fMRI studies (see Vanduffel et al. 2001). Because of its localization, area $\mathrm{CSM}_{\mathrm{PPC}}$ might correspond to the PIP area (Colby et al. 1988; Markov et al. 2014). Even though further studies will be needed to clarify this point, it is tempting to hypothesize that there might be a functional dissociation for 3D processing between this area and its counterpart on the lateral bank of the IPS, the caudal intraparietal area (CIP). Indeed, in a previous monkey fMRI study, Durand et al. (2007) revealed sensitivity to kinetic depth in area PIP and AIP (for which we also found activations in M02) but not in area CIP. Area PIP has also been shown to respond to 3D structure (see Alizadeh et al. 2018). It might therefore play a role in the detection of and interaction with moving objects whereas CIP could be involved in processing 3D orientation and/or 3D features/arrangement of elements (Tsutsui et al. 2002; Durand et al. 2007; Rosenberg et al. 2013). In human, the studies of Likova and Tyler (2007) and of Rokers et al. (2009) did not explore stereomotion selectivity in the parietal cortex (the latter nonetheless reported significant responses to CDOT in dorsal area V3A). The only study that reported results at the whole-brain level (Kaestner et al. 2019) found strong stereomotion responses in area IPS-0, which is located in the caudal part of the human IPS and therefore

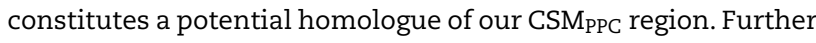
studies will be necessary to clarify this point.

Finally, we did not find CSM-specific activations in area V3A although this area is motion selective in macaque (Galletti et al. 1990). Stereomotion significantly activated human area V3A in previous studies (Likova and Tyler 2007; Rokers et al. 2009; Kaestner et al. 2019). In this area, this is not the only functional difference in motion processing between the two species as responses to structure from motion are known to be much stronger in human V3A than in macaque V3A (Vanduffel et al. 2002, see also Orban et al. 2003).

In order to avoid eye movements, and notably vergence, to contaminate our activations, we took several precautions. As mentioned in the Material and methods section, we only kept runs for which fixation performance was above $85 \%$. Furthermore, our stimuli were designed to avoid driving vergence, with an average disparity value across space that was always equal to zero. Finally, we used the detected saccades as regressors of noninterest in our GLM. It is also worth noting that the activations we observed when contrasting our two conditions of interest (CSM vs. TS) are different from the vergence networks as investigated in terms of vergence tracking and vergence steps by Ward and collaborators (Ward et al. 2015). Additional analyses based on fixation performances and variance of eye position along the $x$ - and $y$-axes during the CSM and TS conditions further demonstrated that eye movements did not impact our results (see Supplementary Fig. S2 and the accompanying text).

\section{Conclusion}

Our fMRI recordings in two macaques demonstrated that CSM is mainly processed by three cortical areas: $\mathrm{CSM}_{\mathrm{STS}}, \mathrm{CSM}_{\mathrm{ITG}}$, and $\mathrm{CSM}_{\mathrm{PPC}}$. We also observed a moderate selectivity in areas of the MT cluster, mostly MSTv, and FST. These results are close to those described in human using a similar experimental protocol and therefore suggest that the cortical network processing stereomotion is relatively well preserved between the two primate species.

\section{Supplementary Material}

Supplementary material is available at Cerebral Cortex online.

\section{Funding}

Agence Nationale de la Recherche Jeunes Chercheuses et Jeunes Chercheurs (grants ANR-16-CE37-0002-01, 3D3M); ATP funding from the Paul Sabatier University awarded to B.R.C.

\section{Notes}

We thank the Inserm/UPS UMR1214 Technical Platform for the MRI acquisitions. Conflict of Interest: None.

\section{References}

Alizadeh AM, Van Dromme I, Verhoef BE, Janssen P. 2018. Caudal intraparietal sulcus and three-dimensional vision: a combined functional magnetic resonance imaging and single-cell study. Neuroimage. 166:46-59.

Backus BT, Fleet DJ, Parker AJ, Heeger DJ. 2001. Human cortical activity correlates with stereoscopic depth perception. J Neurophysiol. 86(4):2054-2068.

Boynton GM, Engel SA, Glover GH, Heeger DJ. 1996. Linear systems analysis of functional magnetic resonance imaging in human V1. J Neurosci. 16(13):4207-4221.

Britten KH, Newsome WT, Shadlen MN, Celebrini S, Movshon JA. 1996. A relationship between behavioral choice and the visual responses of neurons in macaque MT. Vis Neurosci. 13(1):87-100.

Colby CL, Gattass R, Olson CR, Gross CG. 1988. Topographical organization of cortical afferents to extrastriate visual area PO in the macaque: a dual tracer study. J Comp Neurol. 269(3):392-413. doi: 10.1002/cne.902690307.

Cottereau BR, McKee SP, Ales JM, Norcia AM. 2011. Disparitytuned population responses from human visual cortex. J Neurosci. 31(3):954-965.

Cottereau BR, Smith AT, Rima S, Fize D, Héjja-Brichard Y, Renaud L, Durand J-B. 2017. Processing of egomotion-consistent optic flow in the rhesus macaque cortex. Cereb Cortex. 27(1):330-343. 
Czuba TB, Huk AC, Cormack LK, Kohn A. 2014. Area MT encodes three-dimensional motion. J Neurosci. 34(47):15522-15533.

Diedrichsen J, Shadmehr R. 2005. Detecting and adjusting for artifacts in fMRI time series data. Neuroimage. 27(3):624-634.

Dumoulin SO, Wandell BA. 2008. Population receptive field estimates in human visual cortex. Neuroimage. 39:647-660.

Durand JB, Nelissen K, Joly O, Wardak C, Todd JT, Norman JF, Janssen P, Vanduffe W, Orban GA. 2007. Anterior regions of monkey parietal cortex process visual 3D shape. Neuron. 55(3):493-505.

Felleman DJ, Kaas JH. 1984. Receptive-field properties of neurons in middle temporal visual area (MT) of owl monkeys. J Neurophysiol. 52:488-513.

Friston KJ, Fletcher P, Josephs O, Holmes A, Rugg MD, Turner R. 1998. Event-related fMRI: characterizing differential responses. Neuroimage. 7:30-40.

Galletti C, Battaglini PP, Fattori P. 1990. 'Real-motion' cells in area V3A of macaque visual cortex. Exp Brain Res. 82(1):67-76.

Huk AC, Dougherty RF, Heeger DJ. 2002. Retinotopy and functional subdivision of human areas MT and MST. J Neurosci. 22(16):7195-7205.

Joo SJ, Czuba TB, Cormack LK, Huk AC. 2016. Separate perceptual and neural processing of velocity-and disparity-based 3D motion signals. J Neurosci. 36(42):10791-10802.

Kaestner M, Maloney RT, Wailes-Newson KH, Bloj M, Harris JM, Morland AB, Wade AR. 2019. Asymmetries between achromatic and chromatic extraction of 3D motion signals. Proc Natl Acad Sci. 116(27):13631-13640. doi: 10.1073/ pnas. 1817202116.

Kay KN, Winawer J, Mezer A, Wandell BA. 2013. Compressive spatial summation in human visual cortex. J Neurophysiol. 110(2):481-494.

Kolster H, Janssens T, Orban GA, Vanduffel W. 2014. The retinotopic organization of macaque occipitotemporal cortex anterior to V4 and caudoventral to the middle temporal (MT) cluster. J Neurosci. 34(31):10168-10191.

Kolster H, Mandeville JB, Arsenault JT, Ekstrom LB, Wald LL, Vanduffel W. 2009. Visual field map clusters in macaque extrastriate visual cortex. J Neurosci. 29(21):7031-7039.

Kolster H, Peeters R, Orban GA. 2010. The retinotopic organization of the human middle temporal area MT/V5 and its cortical neighbours. J Neurosci. 30(29):9801-9820.

Kriegeskorte N, Simmons WK, Bellgowan PS, Baker CI. 2009. Circular analysis in systems neuroscience - the dangers of double dipping. Nat Neurosci. 12(5):535-540.

Leite FP, Tsao D, Vanduffel W, Fize D, Sasaki Y, Wald LL, Dale AM, Kwong KK, Orban GA, Rosen BR, et al. 2002. Repeated fMRI using iron oxide contrast agent in awake, behaving macaques at 3Tesla. Neuroimage. 16(2):283-294.

Li P, Zhu S, Chen M, Han C, Xu H, Hu J, Fang Y, Lu HD. 2013. A motion direction preference map in monkey V4. Neuron. 78(2):376-388.

Likova LT, Tyler CW. 2007. Stereomotion processing in the human occipital cortex. Neuroimage. 38(2):293-305.

Logothetis NK, Wandell BA. 2004. Interpreting the BOLD signal. Annu Reu Physiol. 66:735-769.

Markov NT, Ercsey-Ravasz MM, Ribeiro Gomes AR, Lamy C, Magrou L, Vezoli J, Misery P, Falchier A, Quilodran R, Gariel $\mathrm{MA}$, et al. 2014. A weighted and directed interareal connectivity matrix for macaque cerebral cortex. Cerebral Cortex. 24(1):17-36. doi: 10.1093/cercor/bhs270.

Maunsell JH, Van Essen DC. 1983. Functional properties of neurons in middle temporal visual area of the macaque mon- key. II. Binocular interactions and sensitivity to binocular disparity. J Neurophysiol. 49:1148-1167.

Maunsell JH, Newsome WT. 1987. Visual processing in monkey extrastriate cortex. Annu Rev Neurosci. 10(1):363-401.

McLaren DG, Kosmatka KJ, Kastman EK, Bendlin BB, Johnson SC. 2010. Rhesus macaque brain morphometry: a methodological comparison of voxel-wise approaches. Methods. 50: 157-165.

McLaren DG, Kosmatka KJ, Oakes TR, Kroenke CD, Kohama SG, Matochik JA, Ingram DK, Johnson SC. 2009. A populationaverage MRI-based atlas collection of the rhesus macaque. Neuroimage. 45:52-59.

Nefs HT, O'Hare L, Harris JM. 2010. Two independent mechanisms for motion-in-depth perception: evidence from individual differences. Front Psychol. 1:155.

Nelissen K, Vanduffel W, Orban GA. 2006. Charting the lower superior temporal region, a new motion-sensitive region in monkey superior temporal sulcus. J Neurosci. 26:5929-5947.

Newsome WT, Paré EB. 1988. A selective impairment of motion perception following lesions of the middle temporal visual area (MT). J Neurosci. 8(6):2201-2211.

Orban GA. 2002. Functional MRI in the awake monkey: the missing link. J Cogn Neurosci. 14(6):965-969.

Orban GA, Fize D, Peuskens H, Denys K, Nelissen K, Sunaert S, Todd J, Vanduffel W. 2003. Similarities and differences in motion processing between the human and macaque brain: evidence from fMRI. Neuropsychologia. 41(13):1757-1768.

Pigarev IN, Nothdurft HC, Kastner S. 2002. Neurons with radial receptive fields in monkey area V4A: evidence of a subdivision of prelunate gyrus based on neuronal response properties. Exp Brain Res. 145(2):199-206.

Pitzalis S, Fattori P, Galletti C. 2013. The functional role of the medial motion area V6. Front Behav Neurosci. 6:91.

Rokers B, Cormack LK, Huk AC. 2009. Disparity-and velocitybased signals for three-dimensional motion perception in human MT+. Nat Neurosci. 12(8):1050.

Rosenberg A, Cowan NJ, Angelaki DE. 2013. The visual representation of 3D object orientation in parietal cortex. J Neurosci. 33(49):19352-19361. doi: 10.1523/JNEUROSCI.3174-13.2013.

Sakata H, Taira M, Kusunoki M, Murata A, Tanaka Y. 1997. The TINS lecture; the parietal association cortex in depth perception and visual control of hand action. Trends Neurosci. 20:350-357.

Sanada TM, DeAngelis GC. 2014. Neural representation of motion-in-depth in area MT. J Neurosci. 34(47):15508-15521.

Shikata E, McNamara A, Sprenger A, Hamzei F, Glauche V, Büchel C, Binkofski F. 2007. Localization of human intraparietal areas AIP, CIP, and LIP using surface orientation and saccadic eye movement tasks. Hum Brain Mapp. 29(4):411-421.

Takeuchi H, Taki Y, Hashizume H, Sassa Y, Nagase T, Nouchi R, Kawashima R. 2011. Effects of training processing speed on neural systems. J Neurosci. 31(34):12139-12148.

Tsutsui K-I, Sakata H, Naganuma T, Taira M. 2002. Neural correlates for perception of 3D surface orientation from texture gradient. Science. 298(5592):409-412. doi: 10.1126/science. 1074128.

Van Essen DC, Drury HA, Dickson J, Harwell J, Hanlon D, Anderson $\mathrm{CH}$. 2001. An integrated software suite for surfacebased analyses of cerebral cortex. J Am Med Inform Assoc. 8(5):443-459.

Vanduffel W, Farivar R. 2014. Functional MRI of awake behaving macaques using standard equipment. In: Dorina Papageorgiou T, Christopoulos GI, Smirnakis SM, IntechOpen, 
editors. Advanced brain neuroimaging topics in health and disease - methods and applications. doi: 10.5772/58281.

Vanduffel W, Fize D, Mandeville JB, Nelissen K, Van Hecke P, Rosen BR, Tootell RB, Orban GA. 2001. Visual motion processing investigated using contrast agent-enhanced fMRI in awake behaving monkeys. Neuron. 32(4):565-577.

Vanduffel W, Fize D, Peuskens H, Denys K, Sunaert S, Todd JT, Orban GA. 2002. Extracting 3D from motion: differences in human and monkey intraparietal cortex. Science. 298(5592):413-415.

Verhoef BE, Bohon KS, Conway BR. 2015. Functional architecture for disparity in macaque inferior temporal cortex and its relationship to the architecture for faces, color, scenes, and visual field. J Neurosci. 35(17):6952-6968.

Wall MB, Smith AT. 2008. The representation of egomotion in the human brain. Curr Biol. 18(3):191-194.

Ward MK, Bolding MS, Schultz KP, Gamlin PD. 2015. Mapping the macaque superior temporal sulcus: functional delineation of vergence and version eye-movement-related activity. J Neurosci. 35(19):7428-7442.

Watanabe M, Tanaka H, Uka T, Fujita I. 2002. Disparity-selective neurons in area V4 of macaque monkeys. J Neurophysiol. 87(4):1960-1973. 